\title{
Resting-state connectivity deficits associated with impaired inhibitory control in non-treatment-seeking adolescents with psychotic symptoms
}

\author{
S. C. Jacobson McEwen ${ }^{1,2}$, C. G. Connolly ${ }^{1,3}$, A. M. C. Kelly ${ }^{4,5}$, I. Kelleher ${ }^{5}$, E. O'Hanlon ${ }^{5}$, \\ M. Clarke ${ }^{5}$, M. Blanchard ${ }^{5}$, S. McNamara ${ }^{1}$, D. Connor ${ }^{1}$, E. Sheehan ${ }^{1}$, G. Donohoe ${ }^{6}$, M. $^{2}$ \\ Cannon ${ }^{5,7,{ }^{*} \text {, and H. Garavan }}{ }^{1,8,{ }^{*}}$
}

${ }^{1}$ School of Psychology, Trinity College Dublin, Dublin, Ireland ${ }^{2}$ Department of Psychiatry \& Biobehavioral Sciences, University of California, Los Angeles, CA ${ }^{3}$ Department of Psychiatry, University of California, San Francisco, CA ${ }^{4}$ Phyllis Green and Randolph Cowen Institute for Pediatric Neuroscience, New York University Child Study Center, New York, NY, USA ${ }^{5}$ Department of Psychiatry and Psychology, Royal College of Surgeons in Ireland, Dublin ${ }^{6}$ Department of Psychiatry \& Neuropsychiatric Genetics Research Group, School of Medicine and Trinity College Institute of Neuroscience, Trinity College, Dublin ${ }^{7}$ Department of Psychiatry, Beaumont Hospital, Dublin, Ireland ${ }^{8}$ Departments of Psychiatry and Psychology, University of Vermont, Burlington, VT, USA

\section{Abstract}

Objective-Psychotic symptoms are common in the population and index risk for a range of severe psychopathological outcomes. We wished to investigate functional connectivity in a community sample of adolescents who reported psychotic symptoms (the extended psychosis phenotype).

\begin{abstract}
Method-This study investigated intrinsic functional connectivity (iFC) during resting-state functional magnetic resonance imaging (fMRI; rs-fMRI). Following screening in schools, 11 nontreatment seeking, youth with psychotic symptoms (aged 11-13) and 14 community controls participated in the study. Seed regions of interest comprised brain regions previously shown to exhibit aberrant activation during inhibitory control in adolescents with psychotic symptoms.
\end{abstract}

\begin{abstract}
Results-Relative to controls, adolescents with psychotic symptoms exhibited reduced iFC between regions supporting inhibitory control. Specifically, they showed weaker iFC between the right inferior frontal gyrus (IFG) and the cingulate, IFG and the striatum, anterior cingulate and claustrum, and precuneus and supramarginal gyrus. Conversely, the psychotic symptoms group exhibited stronger iFC between the superior frontal gyrus and claustrum and IFG and lingual gyrus.
\end{abstract}

Conclusion-The present findings are the first to reveal aberrant functional connectivity in resting-state networks in a community sample of adolescents with psychotic symptoms and suggest that disruption in integration between distributed neural networks (particularly between

(C) 2013 John Wiley \& Sons A/S. Published by John Wiley \& Sons Ltd

Sarah Jacobson McEwen, UCLA Department of Psychiatry \& Biobehavioral Sciences, Semel Institute for Neuroscience and Human Behavior, Office 27-362, 760, Westwood Plaza, Los Angeles, CA 90095, USA. smcewen @ mednet.ucla.edu.

Both authors contributed equally to this work.

Declaration of interest

None. 
prefrontal, cingulate and striatal brain regions) may be a key neurobiological feature of the extended psychosis phenotype.

\section{Keywords}

psychosis; psychotic symptoms; functional magnetic resonance imaging; resting-state; intrinsic functional connectivity

\section{Introduction}

Psychotic symptoms such as hallucinations and delusions are reported at far higher rates in the community than would be expected, given the prevalence of psychotic disorders $(1,2)$. The high prevalence of such symptoms among children and adolescents is particularly striking. A meta-analysis of studies of psychotic symptoms in community-based adult samples revealed a median prevalence of 5\% (3), while a meta-analysis of such studies in children and adolescents revealed median prevalences of $17 \%$ and $7.5 \%$, respectively (4). Individuals who report psychotic symptoms are considered to form part of an 'extended psychosis phenotype', with psychotic disorders at one end of the continuum and isolated psychotic symptoms not associated with dysfunction or distress at the other end $(5,6)$. Longitudinal research has established that young people who report psychotic symptoms are at elevated risk for psychotic disorders $(7,8)$. These findings have led to a great deal of interest in studying young people with psychotic symptoms as putative at-risk model of psychosis - a 'symptomatic risk' group for psychosis, complementary to the familial/genetic high risk (9) and 'ultra high risk'/prodromal $(10,11)$ approaches. More recently, researchers have shown that young people who report psychotic symptoms are at increased risk of a wide variety of disorders, not limited to psychosis (12) with particular high risk for suicidal behaviour $(13,14)$.

While a great deal of epidemiological work has been conducted with young people with psychotic symptoms, neuroimaging research in this population is only now beginning. We recently reported a number of structural and functional neurobiological abnormalities in a community sample of young adolescents reporting psychotic symptoms (15). Specifically, relative to healthy comparison participants, adolescents who reported psychotic symptoms exhibited reduced functional activation during a GO/NOGO response inhibition task within the right prefrontal cortex (PFC), temporal lobe and claustrum, as well as grey matter volume reductions in the inferior temporal lobe and reduced white matter integrity along the inferior longitudinal fasciculus, cingulum bundle and inferior fronto-occipital fasciculus (15). Recent neurocognitive research in a community sample of young people with psychotic symptoms demonstrated marked impairment in processing speed, which the authors suggested may point to aberrant functional connectivity within and between wholebrain neural systems, rather than indexing impairment in discrete neural networks $(16,17)$. Direct evidence for this, however, is lacking.

Resting-state MRI provides a powerful means for studying whole-brain neural connectivity and identification of aberrant intrinsic functional connectivity (iFC) within and between neural systems. Psychotic symptoms among schizophrenia patients have been associated with abnormal iFC between the fronto-occipital network and the anterior default mode network (DMN) and the PFC network (18), revealing the relationship between brain dysconnectivity and its effects on the core pathology of the illness. Reduced iFC has been reported in individuals at genetic high risk for schizophrenia, particularly within the dorsolateral PFC and the DMN (19). Reduced iFC in the DMN has also been reported in siblings of patients with schizophrenia (20). To date, only one relatively small study has examined iFC in a sample of 'ultra high risk' or individuals fulfilling criteria for a 
prodromal syndrome and the authors reported hyperconnectivity within the DMN regions and concluded that abnormal resting-state network activity may be related to the clinical picture of the UHR state (21). There have been no iFC reports to date, however, in the extended psychosis phenotype - that is, in members of the general population who report psychotic symptoms. In this study, we wished to use resting-state functional magnetic resonance imaging (fMRI) to investigate functional connectivity in a community sample of adolescent with psychotic symptoms.

\section{Aims of the study}

We examined resting-state functional magnetic resonance imaging intrinsic functional connectivity in a non-treatment seeking, community-based sample of individuals with psychotic symptoms to investigate 1) whether previously reported task-related regions of neural abnormality also revealed a similar pattern of effect in the underlying functional connectivity and 2) if present, to identify the brain regions with associated these areas. To achieve this, we used a seed region of interest approach with seeds centred on the locations of significant group differences in a response inhibition task activation reported in our previous study. We hypothesised that impaired intrinsic functional connectivity in the psychotic symptoms group would accompany the reported task-related hypoactivity, particularly between cingulo-opercular and fronto-temporal brain regions.

\section{Material and methods}

\section{Design}

Case-control study.

\section{Patients and recruitment}

Adolescents aged 11-13 years who reported psychotic symptoms and comparison adolescents who did not report psychotic symptoms aged 11-13 years were recruited from primary (elementary)-level schools in North Dublin, Ireland. A short, seven-item self-report questionnaire, the Adolescent Psychotic-Like Symptom Screener (APSS) (22), was administered to 277 adolescents in the classroom to screen for the presence of psychotic symptoms. The details of the recruitment and participant selection for this sample can be found in our previous study (15).

A total of 37 of the screened adolescents subsequently participated in a clinical interview with a trained psychiatrist or psychologist using the Psychosis section of the Schedule for Affective Disorders and Schizophrenia for School-Aged Children, Present and Lifetime version, (K-SADS) to verify the presence or absence of psychotic symptoms. Based upon the interview and consensus ratings, 20 adolescents were determined to be controls (Con) and 17 reported psychotic symptoms (PSY) at interview. Twelve subjects declined to be scanned, had contraindications to scanning or participated but did not complete the entire scan, resulting in final samples of 11 adolescents with psychotic symptoms and 14 controls. Prior to scanning, all participants completed a practice session of the GO/NOGO task and the Edinburgh Handedness Inventory, to ensure that all participants were right-hand dominant. All participants were Caucasian, native Irish citizens, and were in 5th or 6th grade in mainstream elementary schools. Table 1 contains a summary of the demographic characteristics of the sample.

The parents of each participant provided signed informed consent, and the adolescents signed an informed assent. The study was approved by the Beaumont Hospital Medical Research Ethics Committee and the Trinity College School of Psychology Research Ethics Committee. 


\section{Measurements}

All scanning was conducted on a Philips Intera Achieva 3.0 Tesla MR system (Best, the Netherlands) equipped with a $30.5 \mathrm{~cm}$ internal diameter head coil. Participants viewed stimuli back-projected onto a screen at the head of the scanner bed viewed through a mirror affixed to the head coil. A high-resolution T1-weighted anatomical MPRAGE axial volume $($ FOV $=230 \mathrm{~mm}$, voxel size: $0.9 \mathrm{~mm} \times 0.9 \mathrm{~mm} \times 0.9 \mathrm{~mm})$ was acquired. Task and restingstate functional data were collected using a T2* weighted echo planar imaging sequence that acquired 32 non-contiguous (10\% gap) $3.5 \mathrm{~mm}$ axial slices covering the entire brain (TE = $35 \mathrm{~ms}, \mathrm{TR}=2000 \mathrm{~ms}$, FOV $=224 \mathrm{~mm}, 64 \mathrm{~mm} \times 64 \mathrm{~mm}$ matrix size). Participants completed two runs of a motor GO/NOGO task, lasting $5 \mathrm{~min}$ and $20 \mathrm{~s}$ per run, as previously reported (8), followed by a resting-state scan. Due to the challenges of imaging a young sample, the resting-state scan was $3 \mathrm{~min}$ and $30 \mathrm{~s}$ long (105 time points).

\section{rs-fMRI analysis}

Resting-state data processing was performed using AFNI and FSL in accordance with standard procedures previously reported (23). Preprocessing comprised slice timing and motion correction, spatial smoothing ( $5 \mathrm{~mm}$ FWHM Gaussian kernel), global mean-based intensity normalisation and temporal bandpass filtering $(0.01<f<0.1)$. FSL's FLIRT was used to register the high-resolution T1 images to the $2 \mathrm{~mm}$ MNI152 template (Montreal Neurological Institute). FLIRT was also used to register each participant's resting-state time series to their high-resolution anatomical images.

To control for physiological fluctuations associated with respiratory and cardiac cycles, as well as other sources of spurious variance, each participant's preprocessed 4-D volume was regressed on nine nuisance covariates, comprising the six motion estimates, a global signal averaged across all brain voxels and signals from white matter and cerebral spinal fluid (CSF). Correction for autocorrelation was performed using FSL's FILM. The white matter and CSF regressors were produced by first segmenting each participant's structural images using the FSL segmentation program, FAST. The segmented images for white matter and CSF were thresholded to ensure a $90 \%$ tissue probability, and an average time series was extracted from all voxels within the mask.

This nuisance signal regression step produced a prewhitened 4-D residuals volume for each participant. This residuals volume was scaled by dividing each voxel's time series by its standard deviation, which removes potential between-group differences in the magnitude of the BOLD signal. Finally, the scaled residuals were transformed into MNI152 standard space at $2 \mathrm{~mm}$ resolution, by applying the previously computed transformation.

\section{Seed selection and creation}

We examined iFC associated with regions that exhibited significant group differences in GO/NOGO task activation in our previous study (8). Specifically, 10 spherical MNI-space seed ROIs (4 mm radius) were created and centred on the centres of mass of brain regions revealing between-group activation differences during successful inhibitions on the GO/ NOGO task (Table 2). For each participant, a time series for each seed ROI was extracted from the 4-D residuals MNI-space volume by averaging the time series across all voxels within the ROI. The extracted time series were then scaled by their standard deviations.

\section{Seed-based functional connectivity analysis}

The FsL program FEAT was used to perform a series of whole-brain multiple regression analyses in which each participant's 4-D scaled native-space residuals volume was regressed on each seed time series. This produced participant-level maps of all voxels that were 
positively and negatively correlated with the seed ROI time series. These correlation images were Fisher- $z$ transformed to produce $z$-value maps and transformed into MNI152 $2 \mathrm{~mm}$ standard space for group-level analyses.

\section{Statistical analysis}

Group-level analyses were accomplished using the Fst program FEAT by means of a mixedeffects ordinary least-squares model. Correction for multiple comparisons was performed at the cluster level using the Gaussian random field theory $(\min Z>2.3$; cluster significance: $P$ $<0.05$, corrected).

\section{Results \\ Demographics}

There were no significant group differences for age at date of scanning, gender, handedness, scholastic ability on the WRAT-4 or GO/NOGO task performance (See Table 1). There was a minor but statistically significant difference between groups for years of education, with the psychotic symptom group having half a year less on average than the control adolescents.

\section{Intrinsic functional connectivity results}

Individuals with psychotic symptoms exhibited reduced positive iFC, particularly within frontal, parietal and midline brain regions compared to individuals who did not report psychotic symptoms (Table 3; Fig. 1a). Specifically, the psychotic symptoms group (PSY) group exhibited reduced iFC between the right inferior frontal gyrus (IFG) seed ROI and rostral anterior cingulate cortex (ACC), bilaterally, as well as with another region in the right IFG, which extended into the middle frontal gyrus (MFG) and anterior insula (Fig. 1ai, ii). The psychotic symptoms group also exhibited reduced iFC between the left claustrum seed (proximal to the dorsal anterior insula) and dorsal ACC, bilaterally (Fig. 1aiii), between the right lentiform seed and right IFG and MFG (Fig. 1aiv) and between the right precuneus seed and the right supramarginal gyrus (Fig. 1av).

Figure $1 \mathrm{~b}$ illustrates the brain regions that emerged as significant in the reverse contrast [psychotic symptoms (PSY) > Con]. On inspection, these regions exhibited weaker negative iFC in the psychotic symptom group relative to controls. Specifically, the control group exhibited negative iFC between the right IFG seed and the left lingual gyrus (Fig. 1bi) and between the left claustrum seed and the right superior frontal gyrus (Fig. 1bii). In comparison, the psychotic symptoms group exhibited slightly positive iFC between IFG and lingual gyrus and weaker negative iFC between claustrum and superior frontal gyrus.

The schematic in Fig. 2 summarises these results and integrates them with our previous findings of group differences in GO/NOGO task-related activation. Regions exhibiting significant group differences in GO/NOGO task-related activation, which formed the seed ROIs for the iFC analysis, are shown in red. Regions exhibiting significant group differences in iFC with the seed regions are indicated in black [left panel: controls > psychotic symptom group; right panel: psychotic symptom group > controls].

As Fig. 2 illuminates, the brain regions that exhibited decreased activity during successful inhibitions in the psychotic symptoms group, relative to controls (IFG, claustrum and lentiform nucleus), also exhibited reduced positive iFC, and suggest the involvement of multiple large-scale networks, including an executive control network (IFG seed with inferior/MFG), the cingulo-opercular or salience network (IFG and claustrum seeds with ACC), cortico-striatal networks (lentiform nucleus seed with IFG). The only exception to 
this pattern was the precuneus seed (a key region known to be part of the DMN), which was hyperactive in the psychotic symptoms group during the task, but showed decreased iFC during rest with the supramarginal gyrus in the psychotic symptoms group, relative to controls.

\section{Discussion}

This study examined whole-brain iFC in adolescents with psychotic symptoms by exploring regions previously identified as exhibiting group differences in activation during a response inhibition (GO/NOGO) task. In individuals with psychotic symptoms, the brain regions that were less engaged during successful inhibitions, relative to controls, including the right IFG, claustrum/dorsal anterior insula and lentiform nucleus (dorsal striatum), also exhibited weaker iFC with functionally related brain regions at rest. Furthermore, the group with psychotic symptoms exhibited abnormal patterns of functional differentiation between brain networks - for example, exhibiting greater functional connectivity between dorsal PFC and the claustrum and regions of visual cortex and the IFG, findings consistent with those reported in a recent meta-analysis of rs-fMRI of schizophrenia and depression disorders (24). During successful inhibitions, the psychotic symptoms group revealed reduced activity in the right IFG and right lentiform nucleus (dorsal striatum) (15). Both regions are considered key components of a network supporting inhibitory control (25). Therefore, both executive function deficits and abnormal perceptual experiences appear to be associated with diminished activity within and functional connectivity between the PFC and striatum. At rest, the psychotic symptoms group exhibited reduced local or short-range connectivity between the IFG and proximal regions encompassing the IFG, MFG and anterior insula regions identified with white matter integrity differences in ultra high risk and first-episode schizophrenia samples (26-28). The psychotic symptoms group also exhibited weaker iFC between the IFG and the functionally related dorsal anterior cingulate, which is known to be activated during response inhibition tasks and plays a role in performance monitoring (29), and between the lentiform nucleus and right IFG and MFG. This pattern of results suggests that disconnection is not just a feature of schizophrenia but is also a feature of the much broader extended psychosis phenotype. Here, we suggest that one key locus of dysfunction is the right IFG and that abnormal function in this area in the psychotic symptoms group disrupts the entire network of functionally connected regions underlying response inhibition. This suggestion is consistent with previous findings of reduced right lateralised PFC activity in patients with psychotic illness, which have prompted hypotheses that dysfunction in this region may be central to the cognitive deficits found in psychosis (30).

In addition to disrupted positive functional connections, the psychotic symptoms group exhibited abnormal patterns of functional connectivity between brain regions that exhibited negative iFC in the control group. For example, the psychotic symptoms group exhibited weakly positive iFC between the right IFG seed and the left lingual gyrus, a component of the visual network, while the same functional connection was negative in controls. This observation echoes previous findings in psychosis patients which revealed increased frontooccipital iFC compared to controls (31). Moreover, a recent rs-fMRI study in adolescents revealed a positive correlation between connectivity with the visual network and schizotypy scores (32). Additionally, in chronic schizophrenia patients, positive symptoms scores on the PANSS have been found to be positively correlated with greater fronto-occipital iFC (18).

The psychotic symptoms group also revealed decreased task-related activity in the claustrum, which is a component of the salience network, a network implicated in coordinating brain regions necessary for sensory processing (24). During rs-fMRI the psychotic symptoms group revealed reduced claustrum seed functional connectivity with the dorsal ACC, which is also a component of the salience functional network. The claustrum 
has been found to be a highly connected brain region and known to be involved in the integration of semantic, linguistic and sensory information (25). The dorsal ACC, which is known as the cognitive subdivision of the ACC, has an important role in executive control and control of directed effort, specifically during performance monitoring $(15,26)$. The dorsal ACC is also closely connected to speech processing regions in humans. A negative relationship was found between the strength of the claustrum and dorsal ACC iFC and psychotic symptoms. It is thus plausible that impaired functional connectivity between these regions may contribute to the misattribution and control of the salience given to perceptual experiences. Furthermore, the rs-fMRI analysis further complements the diffusion tensor imaging findings previously published on this psychotic symptoms group (15) where we found disturbed white matter integrity in the cingulum bundle, which passes through the dorsal ACC, in the PSY group. Williamson and Allman (32) propose a model whereby if the directed effort network fails to be fully engaged with the representational network, then thoughts, feelings or actions may be perceived as belonging to someone else and thoughts may be perceived as auditory hallucinations. This fits with the clinical picture in our young people as the most common symptom reported by young people with psychotic symptoms is generally thoughts heard aloud (also known as Gedankenlautwerden or Echo de al Pensee) (22).

There are some limitations to our study. Due to the difficulty involved in the recruitment of children with psychotic symptoms in the community for an imaging study, the sample size of this study was relatively small (although comparable to CHR iFC studies), and results will require replication in a larger study. Our small sample size may have not allowed for more subtle differences in intrinsic connectivity patterns to survive the stringent statistical thresholds employed in the analyses. It must also be stated that this study focuses on previously reported regions of task-related cognitive abnormality (15). The findings of anomalies in a predetermined region do not rule out anomalies elsewhere, and this has been identified as a possible reason for the inconsistencies in the connectivity literature in schizophrenia (33). Further research is necessary to investigate the presence of compromised functional connectivity beyond the regions explored in this study. A major issue in all imaging studies of psychosis (indeed of any psychiatric disorder) is the issue of approximate replications. It is difficult to find two studies that exactly replicate each other, and this is attributed to differences in measures, analyses, hardware and other unknown factors. A further limitation is that our study did not have sufficient power to allow us to examine whether the effects found were due to psychotic symptoms alone or comorbid psychopathology $(12,14)$. Further research in larger samples, which also include a separate psychopathology group, will allow us to determine which deficits are specific to psychotic symptoms. Finally, studies using advanced imaging techniques such as high angular diffusion weighted imaging will be valuable in yielding more fine-grained data on subtle abnormalities of specific tracts in AR populations.

In conclusion, the present findings are the first to reveal aberrant functional connectivity in resting-state networks in a community sample of individuals with psychotic symptoms. Our results suggest that disruption in integration between distributed neural networks (particularly between prefrontal, cingulate and striatal brain regions) may be a key neurobiological feature of the extended psychosis phenotype.

\section{Acknowledgments}

The research leading to these results has received funding from a Clinician Scientist Award (CSA/2004/1) from the Health Research Board (Ireland) to MC. SJM was funded by an NIH T32 and NIMH K01 Award. EO'H was funded by a Health Research Award (HRA PHS/2012/28) from the Health Research Board Ireland. Funding was also provided by a NARSAD Essel Independent Investigator Award to MC. IK was partly funded by the European Community's Seventh Framework Programme under grant agreement HEALTH-F2-2010-241909 [project 
European Network of National Schizophrenia Networks Studying Gene-Environment Interactions (EU-GEI)]. We acknowledge the use of the facilities of the Clinical Research Centre in the RCSI Education and Research Centre in Beaumont Hospital. We sincerely thank the participants and their parents for giving their time to this study.

\section{References}

1. Kelleher I, Cannon M. Psychotic symptoms in the general population: characterizing a high risk group for psychosis. Psychol Med. 2011; 41:1-6. [PubMed: 20624328]

2. Gale CK, Wells JE, McGee R, Browne MA. A latent class analysis of psychosis-like experiences in the New Zealand Mental Health Survey. Acta Psychiatr Scand. 2011; 124:205-213. [PubMed: 21495982]

3. van Os J, Linscott RJ, Myin-Germeys I, Delespaul P, Krabbendam L. A systematic review and meta-analysis of the psychosis continuum: evidence for a psychosis proneness-persistenceimpairment model of psychotic disorder. Psychol Med. 2009; 39:179-195. [PubMed: 18606047]

4. Kelleher I, Connor D, Clarke M, Devlin N, Harley M, Cannon M. Prevalence of psychotic symptoms in childhood and adolescence: a systematic review and meta-analysis of populationbased studies. Psychol Med. 2012; 9:1-7.

5. Nuevo R, Van Os J, Arango C, Chatterjii S, Ayuso-Mateos JL. Evidence for the early clinical relevance of hallucinatory-delusional states in the general population. Acta Psychiatr Scand. 2012; 127:1-12.

6. Smeets F, Lataster T, van Winkel R, de Graaf R, ten Have M, van Os J. Testing the hypothesis that psychotic illness begins when subthreshold hallucinations combine with delusional ideation. Acta Psychiatr Scand. 2013; 127:34-47. [PubMed: 22676336]

7. Poulton R, Caspi A, Moffitt TE, Cannon M, Murray R, Harrington H. Children's self-reported psychotic symptoms and adult schizophreniform disorder: a 15-year longitudinal study. Arch Gen Psychiatry. 2000; 57:1053-1058. [PubMed: 11074871]

8. Kaymaz N, Drukker M, Lieb R, et al. Do subthreshold psychotic experiences predict clinical outcomes in unselected non-help-seeking population-based samples? A systematic review and metaanalysis, enriched with new results. Psychol Med. 2012; 42:2239-2253.

9. Lawrie SM, Whalley HC, Abukmeil SS, et al. Brain structure, genetic liability, and psychotic symptoms in subjects at high risk of developing schizophrenia. Biol Psychiatry. 2001; 49:811-823. [PubMed: 11343678]

10. Cannon TD, Cadenhead K, Cornblatt B, et al. Prediction of psychosis in youth at high clinical risk: a multisite longitudinal study in North America. Arch Gen Psychiatry. 2008; 65:28-37. [PubMed: 18180426]

11. Rietdijk J, Klaasen R, Ising H, et al. Detection of people at risk of developing a first psychosis: comparison of two recruitment strategies. Acta Psychiatr Scand. 2012; 126:21-30. [PubMed: 22335365]

12. Kelleher I, Keeley H, Corcoran P, et al. Clinicopathological significance of psychotic experiences in non-psychotic young people: evidence from four population-based studies. Br J Psychiatry. 2012; 201:26-32. [PubMed: 22500011]

13. Nishida A, Sasaki T, Nishimura Y, et al. Psychotic-like experiences are associated with suicidal feelings and deliberate self-harm behaviours in adolescents aged 12-15 years. Acta Psychiatr Scand. 2010; 121:301-307. [PubMed: 19614622]

14. Kelleher I, Lynch F, Harley M, et al. Psychotic symptoms in adolescence index risk for suicidal behaviour. Arch Gen Psychiatry. 2012; 69:1277-1283. [PubMed: 23108974]

15. Jacobson S, Kelleher I, Harley M, et al. Structural and functional brain correlates of subclinical psychotic symptoms in 11-13 year old schoolchildren. Neuroimage. 2010; 49:1875-1885. [PubMed: 19770054]

16. Kelleher I, Clarke MC, Rawdon C, Murphy J, Cannon M. Neurocognition in the extended psychosis phenotype: performance of a community sample of adolescents with psychotic symptoms on the MATRICS neurocognitive battery. Schizophr Bull. 2012; 38:1-9. [PubMed: 22102093] 
17. Kelleher I, Murtagh A, Clarke MC, Murphy J, Rawdon C, Cannon M. Neurocognitive performance of a community-based sample of young people at putative ultra high risk for psychosis: support for the processing speed hypothesis. Cogn Neuropsychiatry. 2012; 18:9-25. [PubMed: 22991935]

18. Meda SA, Gill A, Stevens MC, et al. Differences in resting-state functional magnetic resonance imaging functional network connectivity between schizophrenia and psychotic bipolar probands and their unaffected first-degree relatives. Biol Psychiatry. 2012; 71:881-889. [PubMed: 22401986]

19. Whitfield-Gabrieli S, Thermenos HW, Milanovic S, et al. Hyperactivity and hyperconnectivity of the default network in schizophrenia and in first-degree relatives of persons with schizophrenia. Proc Natl Acad Sci USA. 2009; 106:1279-1284. [PubMed: 19164577]

20. Repovs G, Csernansky JG, Barch DM. Brain network connectivity in individuals with schizophrenia and their siblings. Biol Psychiatry. 2011; 69:967-973. [PubMed: 21193174]

21. Shim G, Oh JS, Jung WH, et al. Altered resting-state connectivity in subjects at ultra-high risk for psychosis: an fMRI study. Behav Brain Funct. 2010; 6:58. [PubMed: 20932348]

22. Kelleher I, Harley M, Murtagh A, Cannon M. Are screening instruments valid for psychotic-like experiences? A validation study of screening questions for psychotic-like experiences using indepth clinical interview. Schizophr Bull. 2011; 37:362-369. [PubMed: 19542527]

23. Kelly C, de Zubicaray G, Di Martino A, et al. L-dopa modulates functional connectivity in striatal cognitive and motor networks: a double-blind placebo-controlled study. J Neurosci. 2009; 29:7364-7378. [PubMed: 19494158]

24. Kühn S, Gallinat J. Resting-state brain activity in schizophrenia and major depression: a quantitative meta-analysis. Schizophr Bull. 2013; 39:358-365. [PubMed: 22080493]

25. Kelly AM, Hester R, Murphy K, Javitt DC, Foxe JJ, Garavan H. Prefrontal-subcortical dissociations underlying inhibitory control revealed by event-related fMRI. Eur J Neurosci. 2004; 19:3105-3112. [PubMed: 15182319]

26. Mingoia G, Wagner G, Langbein K, et al. Default mode network activity in schizophrenia studied at resting state using probabilistic ICA. Schizophr Res. 2012; 138:143-149. [PubMed: 22578721]

27. Luck D, Buchy L, Czechowska Y, et al. Fronto-temporal disconnectivity and clinical short-term outcome in first episode psychosis: a DTI-tractography study. J Psychiatr Res. 2011; 45:369-377. [PubMed: 20708198]

28. Peters BD, Szeszko PR, Radua J, et al. White matter development in adolescents: diffusion tensor imaging and meta-analysis results. Schizophr Bull. 2012; 38:1308-1317. [PubMed: 22499780]

29. Garavan H, Ross T, Murphy K, Roche RA, Stein EA. Dissociable executive functions in the dynamic control of behavior: inhibition, error detection, and correction. Neuroimage. 2002; 17:1820-1829. [PubMed: 12498755]

30. Hill K, Mann L, Laws KR, Stephenson CM, Nimmo-Smith I, McKenna PJ. Hypofrontality in schizophrenia: a meta-analysis of functional imaging studies. Acta Psychiatr Scand. 2004; 110:243-256. [PubMed: 15352925]

31. Jafri M, Pearlson G, Stevens M, Calhoun V. A method for functional network connectivity among spatially independent resting-state components in schizophrenia. Neuroimage. 2008; 39:16661681. [PubMed: 18082428]

32. Williamson PC, Allman JM. A framework for interpreting functional networks in schizophrenia. Front Hum Neurosci. 2012; 6:1-14. [PubMed: 22279433]

33. Lagioia A, van de Ville D, Debbané M, Lazeyras F, Eliez S. Adolescent resting state networks and their associations with schizotypal trait expression. Front Syst Neurosci. 2010; 4:35. [PubMed: 20844603] 


\section{Significant outcomes}

- This is the first study to demonstrate functional connectivity abnormalities in a community-based sample of adolescents who report psychotic symptoms.

- The findings suggest that reduced connectivity between right-hemisphere regions in prefrontal-striatal-limbic regions may be related to impaired inhibitory control. 


\section{Limitations}

- Sample size was small and warrants replication in a larger sample.

- Further research is necessary to investigate the presence of compromised functional connectivity beyond the regions explored in this study. 



Fig. 1.

(a) Connectivity maps reveal the brain regions with reduced connectivity among the psychotic symptoms group (PSY) compared to the healthy, control adolescents (Con); (Con $>$ PSY). The seed regions of interest are pictured on the left and were the brain regions which revealed a group difference during successful inhibitions of the GO/NOGO task. The connectivity maps on the right are the brain regions that showed intrinsic functional connectivity with the seed region during rest. (i) Right inferior frontal gyrus (IFG) connectivity with right IFG/middle frontal gyrus (ii) Right IFG connectivity with right rostral anterior cingulate cortex (rACC; iii) Left claustrum connectivity with left dorsal anterior cingulate cortex (dACC; iv) Right lentiform nucleus connectivity with right IFG (v) Right precuneus connectivity with right supramarginal gyrus. (b) Connectivity maps reveal the brain regions with increased connectivity among the psychotic symptoms groups (PSY) compared to the healthy, control adolescents (Con); (PSY > Con). (i) Right IFG connectivity with left lingual gyrus (ii) Left claustrum connectivity with right superior frontal gyrus. 


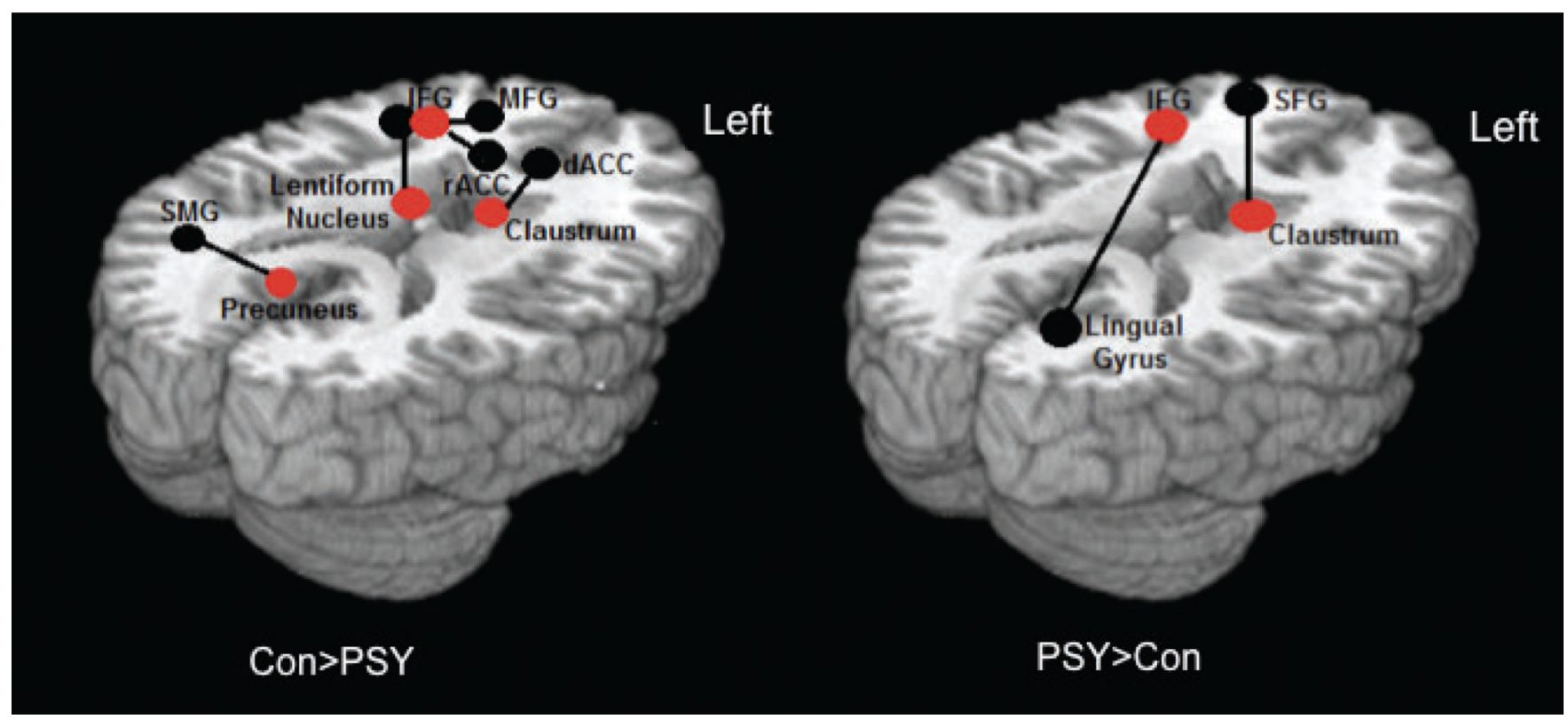

Fig. 2.

Overview of the pathways that showed significant between-group differences in the psychotic symptoms (PSY) and control (Con) adolescents' intrinsic functional connectivity (iFC) analysis. Red dots represent a functionally defined seed ROI derived from the GO/ NOGO task, which were all hypoactive during the task in the PSY group. A black line connects the seed ROI to a black dot, which represents the brain region showing increased functional connectivity with the seed ROI during rest. IFG, inferior frontal gyrus; MFG, middle frontal gyrus; dACC, dorsal anterior cingulate cortex; rACC, rostral anterior cingulate cortex; SMG, supramarginal gyrus; SFG, superior frontal gyrus. 


\section{Table 1}

Characterisation of study participants. Healthy, control adolescents (Con) and the psychotic symptoms (PSY) group

\begin{tabular}{lcc}
\hline & Con $(\boldsymbol{n}=\mathbf{1 4})$ & PSY $(\boldsymbol{n}=\mathbf{1 1})$ \\
\hline Gender $(n, \%)$ & $11(79 \%)$ & $7(64 \%)$ \\
Female & $634(33)$ & $651(22)$ \\
Age (mean weeks, SD) & $11-12$ & $11-13$ \\
Age range (in years) & $5.4(0.5) *$ & $5.9(0.4)$ \\
Education (mean years, SD) & $1(1.4) * *$ & $3(1.1)$ \\
APSS Total Score (out of 7) (mean, SD) & $54(15)$ & $50(5)$ \\
WRAT-4 total reading score (out of 60) (mean, SD) & $42(16)$ & $30(16)$ \\
Per cent correct (mean, SD) & $684(47)$ & $670(90)$ \\
GO/NOGO task GO trial reaction time (mean in ms, SD)
\end{tabular}

CON, control group; PSY, psychotic symptom group; SD, standard deviation; APSS, Adolescent Psychotic-Like Symptom Screener; WRAT, Wide Ranging Achievement Test; ms, milliseconds.

Significant between-group difference

${ }^{*} P=0.01$,

*** $P=0.001$. 


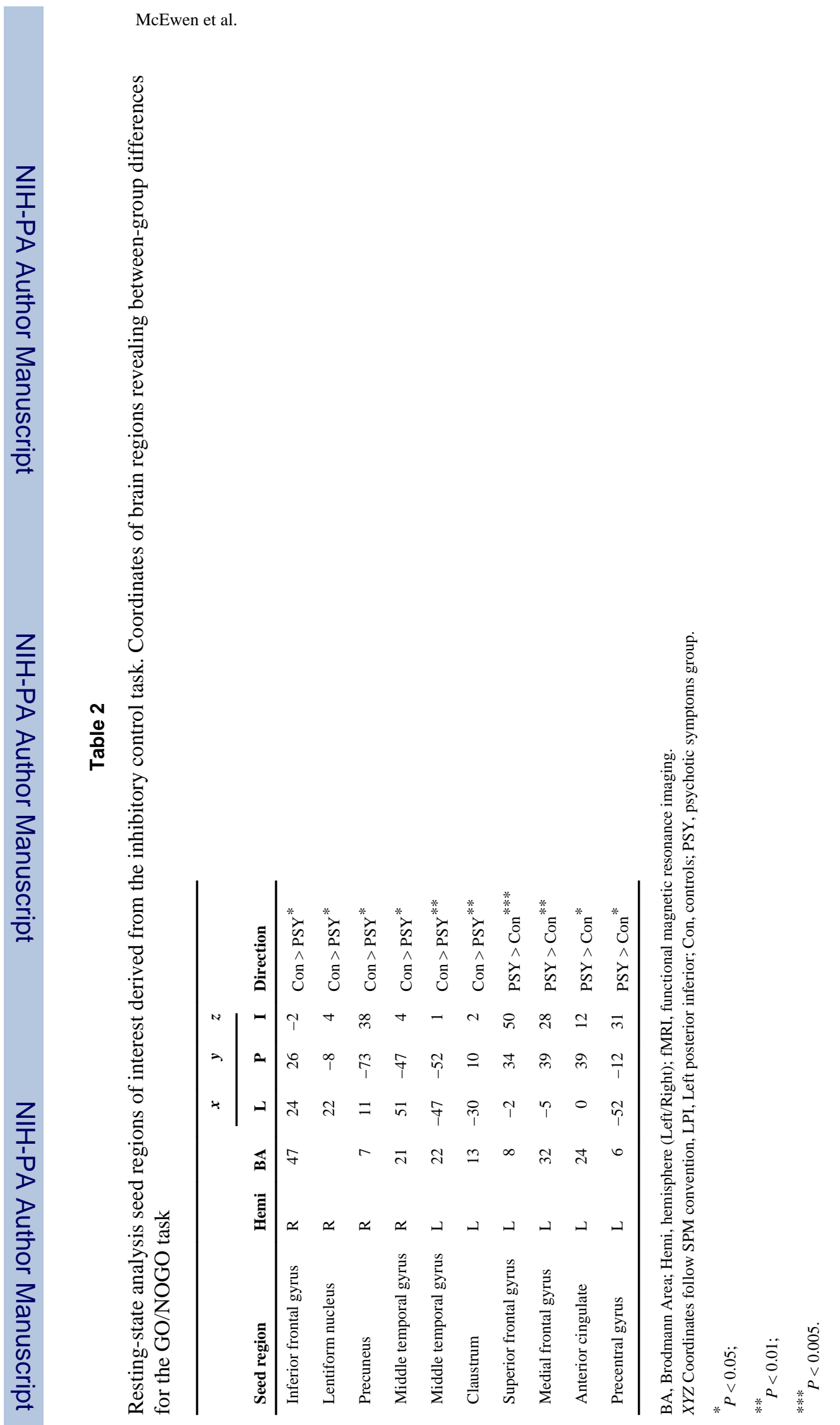

Acta Psychiatr Scand. Author manuscript; available in PMC 2014 February 01. 


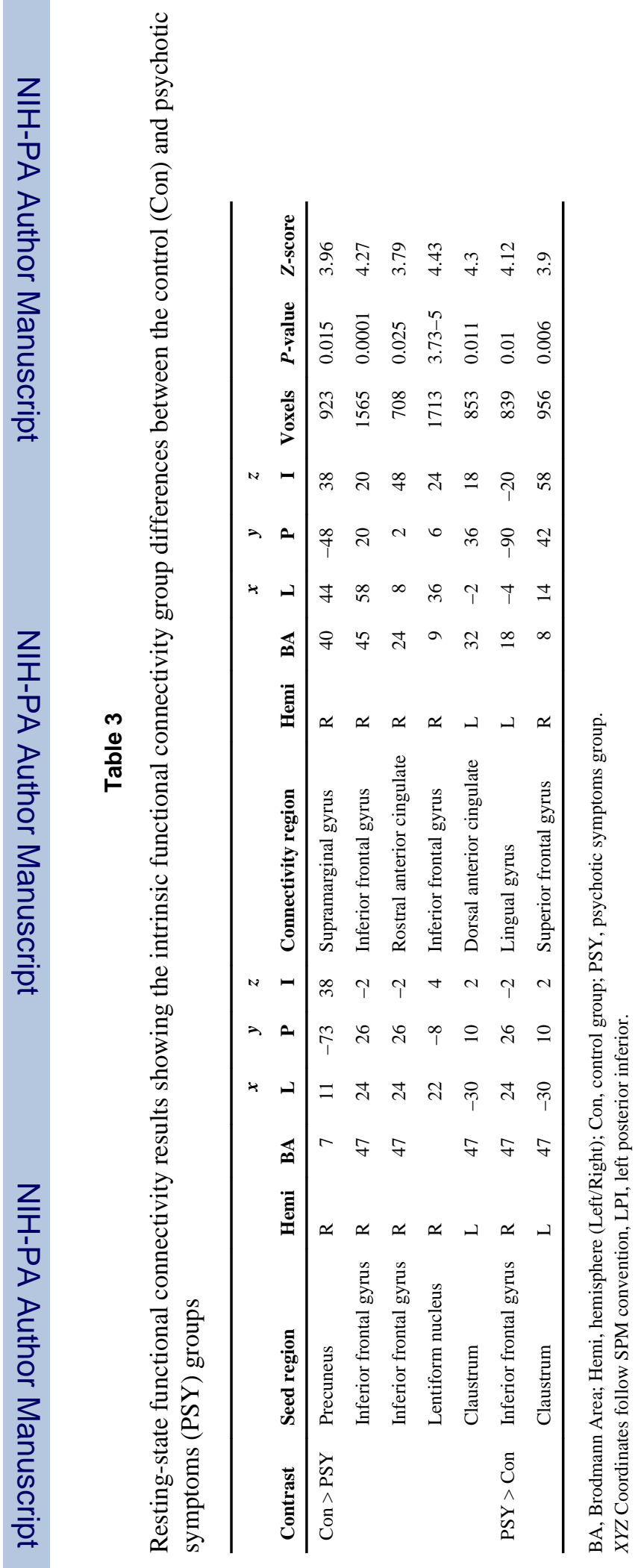

Acta Psychiatr Scand. Author manuscript; available in PMC 2014 February 01. 